\title{
Stellar Photometry with the Optical Monitors
}

\author{
E. Antonello ${ }^{1}$, M. Cropper ${ }^{2}$ \\ ${ }^{1}$ Osservatorio Astronomico di Brera, Via E. Bianchi 46, 22055 Merate, Italy, \\ ${ }^{2}$ Mullard Space Science Laboratory, University College London, Holmbury St Mary, \\ Dorking, Surrey RH5 6NT, UK.
}

\begin{abstract}
The Optical Monitors are small optical telescopes which will fly on board of the X-ray satellites SPECTRUM-X-GAMMA (JET-X experiment) and XMM. Their main scientific applications are the simultaneous observations (imaging) in the optical and UV band of the optical counterparts of X-ray sources, with limiting magnitudes of about $\mathrm{m}_{V}=22$ (JET-X) and 24 (XMM). The OMs are developed in order to perform also serendipitous observations with high photometric precision of bright stars falling in the field of view, which should allow the detection of stellar microvariability at a level better than $10^{-5}$. The main characteristics of the instruments are presented and the problems to be solved in order to reach the scientific goals are briefly discussed.
\end{abstract}

\section{Introduction}

The project of an Optical Monitor (OM) for X-ray satellites derives from the scientific need of having complete data coverage at various wavelengths, UV and optical, of the observed sources, from AGN to bright stars. In principle, optical observations simultaneous with $\mathrm{X}$-ray ones can be performed from ground based telescopes and indeed this procedure has been adopted thus far. However, the complexity of satisfying the constraints typical of the optical telescopes (weather conditions, source observability) and of the X-ray instrumentation (e.g. orbital constraints) lead inevitably to a substantial loss of observing time, both with the optical and X-ray instrument. Therefore, the only practical way of having at the same time an optimal utilization of the time available for $\mathrm{X}$-ray observations, and the wealth of simultaneous UV-optical observations, is to have a small telescope, co-aligned to the X-ray one, as part of the mission. Other advantages of the OM are the immediate identification of the optical counterparts of X-ray sources, mainly in crowded fields, and the possibility of performing a continuous monitoring for long time intervals (days). The latter opportunity along with the provided high precision of differential photometry measurements can be exploited for detecting in serendipity mode very small regular oscillations (microvariability) of bright stars falling in the field of view. This provides an opportunity to develop the research field of asteroseismology. 
Table 1: Main characteristics of the OMs

\begin{tabular}{|c|c|c|}
\hline & JET-X - OM & $\mathrm{XMM}-\mathrm{OM}$ \\
\hline Satellite & $\begin{array}{l}\text { SPECTRUM-R-G (Russia), } \\
\text { JET-X Exper. (UK, Italy, } \\
\text { Germany, Russia, ESTEC) }\end{array}$ & XMM (ESA) \\
\hline Launch date & 1995 & 1999 \\
\hline Orbit & 4 days & 1 day \\
\hline Lifetime & $>3$ years & $>10$ years \\
\hline $\begin{array}{l}\text { OM Resp. } \\
\text { Status }\end{array}$ & $\begin{array}{l}\text { Italy } \\
\text { Phase C }\end{array}$ & $\begin{array}{l}\text { UK, Italy, USA, Belgium } \\
\text { Phase B }\end{array}$ \\
\hline $\begin{array}{l}\text { Telescope } \\
\text { T3 }\end{array}$ & $\begin{array}{l}\text { Ritchey-Chretien } 26 \mathrm{~cm} \\
\text { f/ } 10.9 \\
\text { mirror with central hole }\end{array}$ & $\begin{array}{l}\text { Ritchey-Chretien } 30 \mathrm{~cm} \\
\text { f/13 } \\
\text { dichroic: } 150-550, \\
\quad 550-1100 \mathrm{~nm}\end{array}$ \\
\hline $\begin{array}{l}\text { First beam } \\
\text { Filter wheel }\end{array}$ & $\begin{array}{l}\text { UV, V, white, stop } \\
\text { 8', pix. size } 1.67 " \\
\text { CCD frame transfer (UV) }\end{array}$ & $\begin{array}{l}\text { UV1, UV2, UV3, U, B, } \\
\text { V, HeII, white, grism1, } \\
\text { magnifier, grism2 } \\
22 \text { ', pix. size 1" } \\
\text { large format MCP device }\end{array}$ \\
\hline $\begin{array}{l}\text { Second beam } \\
\text { Filter wheel }\end{array}$ & $\begin{array}{l}30, \text { pix. size } 6.2 " \\
\text { CCD frame transfer }\end{array}$ & $\begin{array}{l}\mathrm{V}, \mathrm{R}, \mathrm{I}, \mathrm{Z}, \mathrm{H} \alpha \text {, white, } \\
\text { grism1, magnifier, blur, } \\
\text { grism2, stop } \\
30^{\prime}, \text { pix. size } 1.8^{\prime \prime} \\
\text { large CCD, frame transfer }\end{array}$ \\
\hline
\end{tabular}

The present contribution contains a short description of the capability of the OM for the X-ray satellite missions SPECTRUM-X-GAMMA and XMM as regards the scientific applications related to stellar photometry.

\section{The Optical Monitors}

The main characteristics of the OMs are reported in Table 1 and in the schematic layout shown in Figure 1. Conceptually, the OM is a small Ritchey-Chretien telescope with two detectors: one is dedicated mainly to the imaging/photometry with good resolution for a small field (SF) of view in particular in the UV band, and the other mainly to the imaging/photometry with lower resolution for a wide (WF) field around the small one. JET-X OM will make only wide band photometry in few filters, while with XMM OM it will be possible to use several filters, grisms (for low resolution spectroscopy) and a magnifier.

In order to secure the high quality of the photometric data it is essential that 
the spacecraft be well stabilized, or at least a mechanism must be available for the correction of its instability. The reason for this is the nonuniformity of the CCD responsivity within each pixel; this cannot be corrected for by means of flat field techniques. In the case of JET-X OM the original project included a stabilization mechanism and a derotating system (Antonello et al., 1990) which were later removed. Since the star tracker capability of the satellite is limited, the present project foresees the use of the OM itself as an accurate star tracker for satisfying the requirements of pointing stabilization. Similarly, the high photometric precision with the CCD in $\mathrm{XMM} O \mathrm{OM}$ requires a high stability, and in this case the image stability against the drift of the satellite will be guaranteed by a mechanism based on a double-wedge system placed before the CCD. Both in JET-X and in XMM case, the signal needed for the stabilization is derived from the stars observed with one of the two detectors.

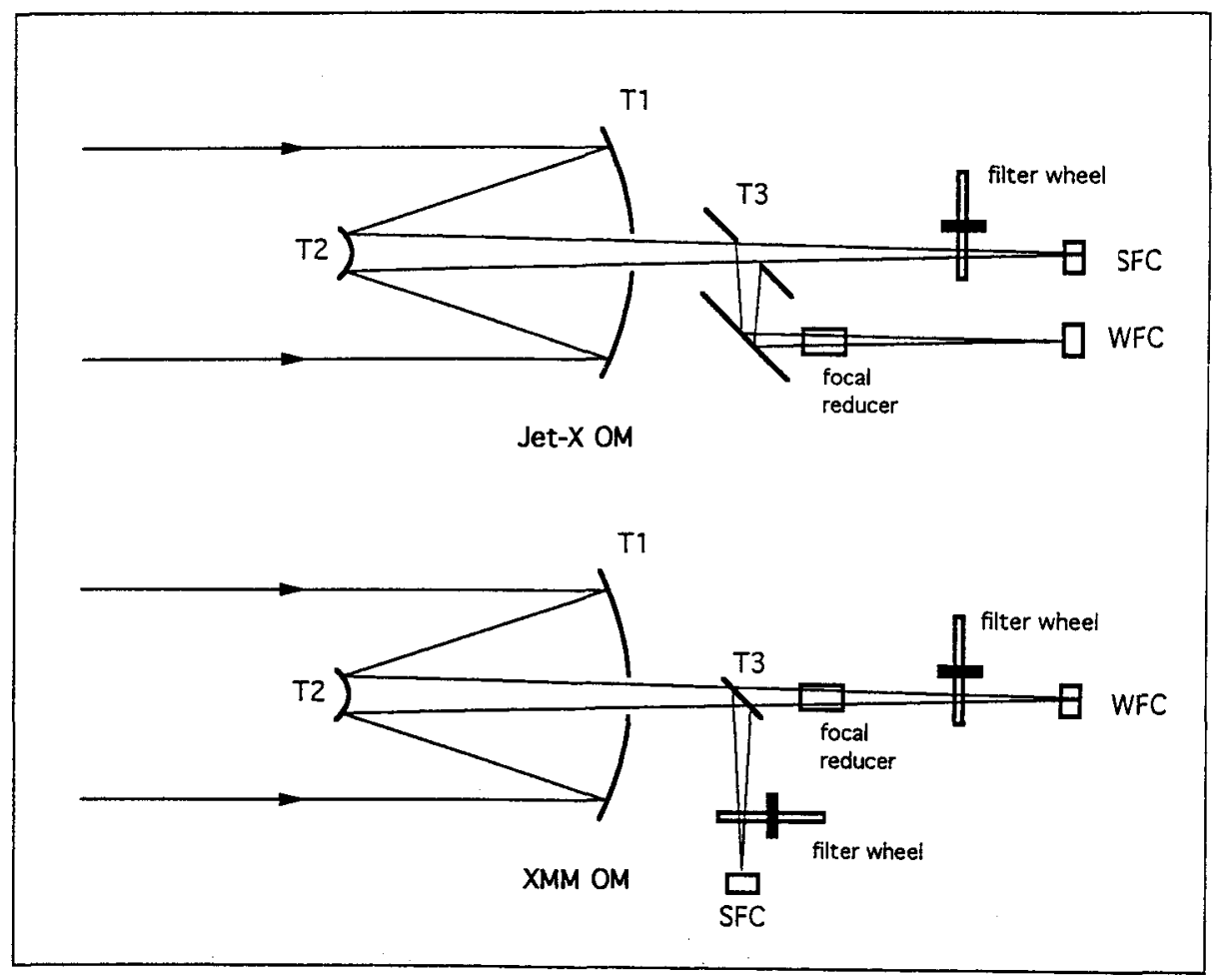

Figure 1. Schematic layout of the OMs

The main difference between JET-X and XMM OM is the detector used for observing the sources in the SF. In JET-X case it is a CCD, UV enhanced, while in XMM case it is a large format photon-counting detector based on a multi-channel plate and CCD readout system (MIC detector). In addition, the red detector in the 
$\mathrm{XMM} O \mathrm{OM}$ is a large format $\mathrm{CCD}$. In view of the large field of MIC, the term SF is somewhat of a misnomer preserved for comparison with the JET-X OM.

\section{JET-X OM}

In JET-X OM a drilled mirror separates the optical beam in two parts. The central part is focussed on the SF focal plane after passing through a changing filter mechanism with three positions, while the surrounding annulus is reflected and focussed on the WF focal plane by a focal reducer. The detector for the SF is a frame transfer UV enhanced CCD TH7863. The main task of the WF camera (WFC, where the detector is another frame transfer CCD TH7863 but not UV enhanced) is to perform the star tracking (with defocussed stellar images) for the stabilization. The working temperature of the two detectors is about $-100^{\circ} \mathrm{C}$ and it is kept at that level by means of a passive radiator.

The CCD frames must be corrected for cosmic ray contamination, and the cosmic ray filtering is performed on board (see Antonello et al., 1990). With the SFC it should be possible to detect faint sources with $\mathrm{m}_{V} \sim 23(\mathrm{~S} / \mathrm{N} \geq 3)$ with integration times of about a thousand seconds. Since the bit rate allotted to OM is limited $(0.5$ lsbit $s^{-1}$ ), there are some constraints on the size and/or the number of the frames telemetred to the ground. As regards the brightest stars falling in the field of view $\left(m_{V} \geq 4\right)$, they are observed by the WFC and used for the star tracking. A total of some tens of bright stars are selected in a threshold mode, and each stellar image is contained within few pixels (e.g. $3 \times 3$ pixels). A series of $1 \mathrm{~s}$ (or less) exposures are summed for a total of several tens of seconds of exposure time, and the subimages corresponding to the selected bright stars are telemetred.

\section{XMM OM}

In XMM OM the beam separator is a dichroic, because the MIC detector in the SFC is dedicated to the observation in the UV-blue band and the CCD to the observation in the red-near IR band. The blue beam is reflected and reaches the SFC after passing through the blue filter wheel, while the red beam is transmitted and reaches the WFC after passing through the image stabilization mechanism, the focal reducer and the red filter wheel. The expected angular resolution on the SFC is 2 arcsec, and it can be about 0.5 arcsec when using the magnifier placed on the filter wheel (working in white light).

With the SFC it is expected to detect a point source as faint as 24th mag with $\mathrm{S} / \mathrm{N}=5$ in $1000 \mathrm{~s}$. Moreover, relatively bright stars $\left(\mathrm{m}_{V} \geq 13\right.$, in the $\mathrm{V}$ filter) can be observed with a time resolution of 0.01 second, and this capability renders this OM suitable for studying phenomena with very short time scales, such as transients. With the WFC it will be possible to study sources brighter than about $\mathrm{m}_{V}=23$ with reasonable exposure times, and it will be possible to observe stars as bright as $m_{V} \sim 4$ using the blurrer placed on the filter wheel for defocussing, and exposure times of few tenths of a second as in JET-X OM case. 
Table 2: Limiting magnitude in white light for different source spectra for detection with XMM OM at a significance of $5 \sigma$ in $1000 \mathrm{~s}$; a, b and $\mathrm{c}$ indicate best, typical and worst background, respectively.

\begin{tabular}{||l|ccc|ccc||}
\hline & \multicolumn{3}{|c|}{ blue beam } & \multicolumn{3}{c||}{ red beam } \\
source & a & b & c & a & b & c \\
\hline B0 star & 24.6 & 24.4 & 23.6 & 23.3 & 23.1 & 22.6 \\
A0 star & 23.6 & 23.4 & 22.5 & 23.6 & 23.4 & 22.6 \\
M0 star & 22.4 & 22.2 & 21.3 & 24.7 & 24.6 & 23.8 \\
\hline
\end{tabular}

The allotted bit rate to this $O M$ is $2 \mathrm{kbit} / \mathrm{s}$, but the expected large amount of data mainly from the MIC detector requires a substantial data compression on board. This data compression will be performed partly using windows and binning techniques instead of full frame images. In view of the 10 years planned lifetime of XMM, substantial effort has been directed at the optimization of the operating modes of the instrument to maximize its capability without introducing unnecessary complexity.

\section{Stellar Photometry}

The expected integration times required to detect a point source using the XMM$\mathrm{OM}$ are reported in Table 2 for a sample of sources and observational conditions. However, apart from the advantage over ground based instruments of observing in the UV band, here probably it is worthwhile to stress on the other advantage of observing from space, that is the possibility of avoiding the limitations given by the earth atmosphere in terms of scintillation and transparency fluctuations which are more important for the brightest stars.

The differential photometry precision for the bright objects is reported in Table 3. The $1 \sigma$-precision has been estimated for a source temperature of $5770 \mathrm{~K}$. Owing to the brightness of the stars, the single exposures are assumed very short, $1 \mathrm{~s}$ or less, and the star image is spread over 4 or more pixels. In this way it is possible to observe bright stars up to the 4th magnitude without saturation and with a conversion factor from electrons to ADU close to the unity. The precisions have been estimated for a cumulative number of exposures of $30 \mathrm{~s}$. In general, the effects of readout noise due to the very high cumulative number of exposures are not significant for very bright stars. As shown in Table 3, the expected minimum detectable amplitude of a sinusoidal variation of the stellar luminosity for an observing time of 3.5 days (JET-X case) can be of few $10^{-6} \mathrm{mag}$, that is close to the amplitude of oscillations of the Sun detected by ACRIM on SMM. As a comparison, we recall that the logarithm of the noise power density at a frequency of about $12 \mathrm{mHz}$ of the photometric measurements is about -4.1 for a ground based $1 \mathrm{~m}$ telescope, -5.3 for the best CCD observations made with a ground based $4 \mathrm{~m}$ telescope (Frandsen, 1992, private communication) and -6.5 for a star with $\mathrm{m}_{V}=5$ observed with the OM. The reason for the improvement 
Table 3: Differential photometry precision (mag) for a cumulative number of 30 exposures of $1 \mathrm{~s}$ of bright stars with $\mathrm{T}=5770 \mathrm{~K}$, and minimum detectable $(99 \%$ confidence level) amplitude of a sinusoidal variation of luminosity for an observing time of 3.5 days (see text).

\begin{tabular}{||c|c|c||c|c|c||}
\hline $\mathrm{m}_{V}$ & precision $(1 \sigma)$ & ampl. & $\mathrm{m}_{V}$ & precision $(1 \sigma)$ & ampl. \\
\hline 4.0 & $6.1 \mathrm{E}-5$ & $4.2 \mathrm{E}-6$ & 8.0 & $3.9 \mathrm{E}-4$ & $2.6 \mathrm{E}-5$ \\
5.0 & $9.8 \mathrm{E}-5$ & $6.6 \mathrm{E}-6$ & 9.0 & $6.2 \mathrm{E}-4$ & $4.2 \mathrm{E}-5$ \\
6.0 & $1.6 \mathrm{E}-4$ & $1.1 \mathrm{E}-5$ & 10.0 & $1.0 \mathrm{E}-3$ & $6.8 \mathrm{E}-5$ \\
7.0 & $2.4 \mathrm{E}-4$ & $1.7 \mathrm{E}-5$ & 11.0 & $1.7 \mathrm{E}-3$ & $1.1 \mathrm{E}-4$ \\
\hline
\end{tabular}

which can be obtained with the OMs is the observation from space, which avoids the atmospheric scintillation and transparency changes.

The precisions reported in Table 3 have been estimated assuming a perfect stabilization of the images and of CCD temperature. Moreover, no contamination owing to cosmic rays and no degrading of the CCD performances due to radiation have been taken into account. The effects of a small wandering of the stellar images on the expected precision have been studied by means of simulations (Antonello, Poretti; this Colloquium) and will be tested in laboratory. It will be possible to correct for the cosmic ray contamination by means an on board frame comparison technique only the images of faint sources with relatively long exposure times, and it will not be possible to apply a similar correction to bright star images; therefore, we expect an incidence of about few percent of time series data contaminated by cosmic rays in the case of bright star observations.

\section{CONCLUSION}

In the present note we have discussed the main characteristics and expected photometric capabilities of the OMs for $\mathrm{X}$-ray satellites, in particular the high precision photometry in the case of observed bright stars. While the rationale for incorporating an optical/UV telescope on the JET-X and XMM spacecraft is driven by a wide variety of needs, the unique position and capabilities of these instruments will open a new window on the study of microvariability and low level pulsations in a large number of stars. This will allow the spectrum of variability to be explored for a variety of stellar masses and evolutionary phases with the promise of a wealth of observational tests of our understanding of stellar structure.

\section{References:}

Antonello E., Citterio O., Mazzoleni F., Mariani A., Pili P., Lombardi P.: 1990, in SPIE Proceedings Conference 1235 on Instrumentation in Astronomy VII, 867. 